\title{
Note sur la nidification dans le sol de quatre Hoplitis KLUG du sous-genre Annosmia WARNCKE (Apoidea, Megachilidae, Osmiini)
}

\section{Par Gérard LE GofF *}

\begin{abstract}
Note on the nest building by Hoplitis of subgenus Annosmia. I provide an overview of the main characteristics of this subgenus with a list of the known species. These bees built their nest in the soil. I then describe the nests of $H$. annulata annulata LATREILLE, $H$. annulata corsaria WARNCKE (with details about the female), $H$. annulata crenulata MORAWITZ, and H. tkalcuella LE GOFF..
\end{abstract}

Résumé. Note sur la nidification d'Hoplitis du sous-genre Annosmia. Je fournis ici une synthèse des caractéristiques de ce sous-genre, avec la liste des espèces connues. Ces abeilles nichent dans le sol. Je décris ensuite la nidification des $H$. annulata annulata LATREILLE, H. annulata corsaria WARNCKE (avec des détails distinctifs de la femelle), H. annulata crenulata MORAWITZ et $H$. tkalcuella LE GOFF.

Mots-clés. Megachilidae, Osmiini, Hoplitis, Annosmia, nidification.

\section{Introduction}

Le sous-genre Annosmia WARNCKE 1991 est très proche du sbg. Hoplitis s. str. Comme elles, ces petites abeilles butinent aussi différentes espèces d'Echium (Boraginaceae). C'est Warncke (1991) qui a distingué ce groupe d'abeilles des autres Hoplitis; van der Zanden (1991) a également publié des descriptions d'Annosmia, mais la plupart de ces espèces se sont révélées être synonymiques de celles décrites par Warncke (1991).

Ces abeilles hoplitiformes sont de petite taille (5-11 mm). Les femelles ont les mandibules tridentées. Les tergites sont bordés de bandes de poils clairs. Le clypéus est le plus souvent dentelé. Les mâles ont également les mandibules tridentées (bidentées chez Hoplitis s.str.) ; le $6^{\text {ème }}$ tergite est simple, avec 2 dents latérales, le $7^{\text {ème }}$ est bifide (ce qui est la principale caractéristique immédiatement visible chez les mâles).

Dans la liste de Warncke (1991), H. persica a les mandibules bidentées et présente quelques différences avec le reste du sous-genre; le mâle présente aussi un $6^{\text {ème }}$ sternite différent, l'espèce est désormais classée dans le sous-genre Coloplitis GRISWOLD. Michener (2007) n'inclut pas H. marchali PÉREZ dans le sbg. Annosmia mais cite cette Hoplitis s.str. car sa carène marginale au $6^{\text {ème }}$ sternite est peu marquée alors qu'elle est nette chez les autres femelles d'Hoplitis s.str. Ce rebord n'existe pas chez les Annosmia, d'où possibilité de confusion pour les femelles; mais le mâle d'H. marchali a le $7^{\text {ème }}$ tergite entier et non bifide comme tous les mâles d'Annosmia. Une mauvaise lecture de Michener (2007) a mené certains auteurs à intégrer marchali dans les Annosmia, c'est qui est à l'évidence une erreur. D'autre part, H. infrapicta COCKERELL, 1916, d'Afrique du sud,

* 44 rue Albert Malet, F-76360 Barentin, France. E-mail : legoff.gerard@free.fr est vraiment douteuse ; d'ailleurs cette Hoplitis n'a pas encore trouvée sa place dans un sbg. défini. Griswold précise bien que le sbg. Annosmia est paléarctique et que sa limite méridionale est le Soudan.

Le sous-genre Annosmia comprend donc actuellement 30 espèces connues :

annulata LATREILLE, 1811 (annulata annulata LATR., annulata corsaria WARNCKE 1991, annulata crenulata MOR. 1871) ; aqabaensis WARNCKE, 1991 ; bassana WARNCKE, 1991 ; cercomela WARNCKE, 1991 ; christae WARNCKE, 1991 ; chukar WARNCKE, 1991 ; elaziga WARNCKE, 1991 ; eremophila WARNCKE, 1991 ; fulica WARNCKE, 1991 ; gentilis WARNCKE, 1991 ; gusenleitneri WARNCKE, 1991 ; hiericonica MAVROMOUSTAKIS, 1949 (hierichonica hiericonica MAVROMOUSTAKIS 1949, hiericonica wolfi WARNCKE 1991) ; idaensis WARNCKE, 1991 ; israelica WARNCKE, 1991 ; kasabi TKALCU, 2000 ; lefeberi VAN DER ZANDEN, 1991 ; leiocephala MAVROMOUSTAKIS, 1954; mutica WARNCKE, 1991 ; nisa WARNCKE, 1991 (= speculumoides VAN DER ZANDEN 1991); ochruros WARNCKE, 1991 (ochruros crecca WARNCKE 1991, ochruros ochruros WARNCKE 1991) ; parana WARNCKE, 1991 ; peralba VAN DER ZANDEN, 1992 ; samarkanda WARNCKE, 1991 ; segura WARNCKE, 1991 ; sordida BENOIST, 1929 ; speculum BENOIST, 1991 ; tkalcuella LE GOFF, 2003 ; tunica WARNCKE, 1991 ; uncaticornis STANEK, 1969 (uncaticornis cursoria WARNCKE, uncaticornis uncaticornis STANEK 1969) ; verhoeffi MAVROMOUSTAKIS, 1954 ; zonalis PÉREZ, 1895 (zonalis bodenheimeri MAVROMOUSTAKIS, zonalis zonalis PÉREZ, 1895).

Pour B. Tkalcu (comm. pers. 2002), H. annulata LATREILle, 1811 et $H$. crenulata MORAWITZ, 1871 étaient deux espèces distinctes à distribution allopatrique. Warncke (1977), considérant Heriades comme sous-genre d'Osmia, avait rejeté le terme de crenulata, à cause de l'existence d'Heriades crenulatus 
NYLANDER, 1856 et avait nommé l'espèce annulata cretica (Pr B. Tkalcu, comm. pers. 2002). Cependant, ces deux Hoplitis étant tellement proches morphologiquement, il est légitime de considérer que crenulata n'est, comme l'a fait Warncke, qu'une sousespèce d'annulata ; " crenulata » primant sur « cretica», l'espèce est donc maintenant nommée $H$. (Annosmia) annulata crenulata MORAWITZ.

L'espèce $H$. annulata annulata LATREILLE se retrouve à la fois en France et en Espagne. Ajoutons $H$. annulata corsaria WARNCKE (limitée à la Corse) et $H$. tkalcuella LE GOFF, 2003 (pour l'Espagne). Une classification incluant parfois encore les genres Heriades, Chelostoma et surtout Hoplitis comme sous-genres d'Osmia a toujours cours pour certains entomologistes d'Europe continentale. Cela occasionne bien des confusions pour ceux qui abordent la tribu des Osmiini. Heureusement, les travaux de Griswold \& Michener (1998), Ulgricht et al. (2008) et de Müller (2010) s'avèrent fondamentaux et clarifient enfin la classification des Osmiini paléarctiques.

\section{Description de certains nids d'Hoplitis (Annosmia)}

Les taxons concernées par cet article sont :

\section{- H. (A.) annulata LATREILLE, 1811 :}

ssp. annulata LATREILLE : espèce ouest-méditerranéenne. ssp. corsaria WARNCKE, 1991 : espèce limitée à la Corse. ssp. crenulata MORAWITZ, 1871 : espèce méditerranéenne moyenne et orientale.

- H. (A.) tkalcuella LE GOFF, 2003 : espèce ibérique.

\section{H. (A.) annulata ssp. annulata LATREILLE}

Cette sous-espèce a une distribution méditerranéenne occidentale. Le matériel décrit cidessous a été récolté à Almuñecar (Granada) (E) et est conservé dans ma collection personnelle (Nid N560 : fondatrice $\mathrm{N}^{\circ} 13061$ - collecte du 10.v.07 ; Nid N562 : fondatrice $\mathrm{N}^{\circ} 13087$ - collecte du 11.v.07 ; Nid N563 fondatrice $\mathrm{N}^{\circ} 13088$ - collecte du 11.v.07; Nid N564 fondatrice $\mathrm{N}^{\circ} 13089$ - collecte du 11.v.07). Les visites florales ont été étudiées localement sur Echium plantagineum L. (Boraginaceae).

Charles Ferton (1893) est le premier à avoir décrit des nids d'H. annulata annulata, sous le nom d'Osmia crenulata:

"Les environs de Marseille m'ont donné une osmie nouvelle pour notre faune, et dont la nidification n'a pas encore été observée. (...) je n'ai trouvé son nid que quatre fois, et toujours dans le même état d'avancement : contenant une cellule presque terminée. L'insecte nidifie dans un terrain horizontal et argileux, au fond d'une galerie verticale, profonde de six à sept centimètres. Le conduit se termine par une cellule ovale, que la bête approvisionne d'un miel violet, homogène et presque liquide (...) La paroi de la loge, probablement enduite d'un mucilage, est lisse. Fe n'ai pas vu l'osmie creuser elle-même son terrier; je crois cependant pouvoir avancer qu'elle le fait, à cause de l'identité pour la forme et la profondeur des nids que j'ai trouvés, et parce que le sol était vierge au-dessous d'eux."

Cette description montre les qualités indiscutables d'observateur de ce grand entomologiste. Les nids examinés par Ferton (1893) se trouvaient certainement dans une même zone et à l'évidence dans un sol facile à forer. Cette image de nid unicellulaire a été reprise au cours du temps par d'autres auteurs. L'H. annulata annulata n'est pas rare, mais localisée, comme c'est souvent le cas pour beaucoup d'Osmiini. La nature du terrain joue beaucoup sur l'aspect des nidifications, surtout quand le forage rencontre des obstacles. La zone que j'ai prospectée à Almuñecar se trouve au pied de falaises de schistes et carbonates (Figure 1A). Ce terrain est une friche où $E$. plantagineum est abondant et très touffu. J'y ai trouvé une impressionnante diversité d'Hoplitis s.tr. qui nichent dans les talus et dans la roche même. $H$. annulata annulata, elle, nichait dans le sol, en petit nombre, sur une surface de quelques mètres carrés, en bordure des massifs d'Echium vers le pied de la falaise.

L'abeille est très rapide quand elle rentre dans son nid ; il faut être patient et attendre quelques allers et venues pour en localiser l'entrée ; d'autant plus que celle-ci se trouve souvent sous des végétaux ou même des cailloux. Ma grande surprise a été la découverte d'un premier nid (réf. N560, Figures 1A-G) dont l'entrée était protégée par une pierre. Tout le nid était foré en oblique, contre un morceau de pierre plat enfoui en biais dans le sol, mais surtout, cette nidification comportait 4 cellules et des cloisonnements. Au bout d'une première branche, on découvre 2 cellules complètes et cloisonnées (Figure 1D, 1-2) comportant une pâtée pollinique et un œuf à la surface. Cette galerie est fermée au niveau du forage d'accès. De là, une deuxième banche a été creusée, avec encore 2 nouvelles cellules : l'une d'entre elles est complète et close (Figure 1D, 4) l'autre est en approvisionnement (Figure 1D, 3). Un autre nid plus « classique » a ensuite été découvert, il était unicellulaire, mais son forage vertical, qui n'atteignait que $28 \mathrm{~mm}$ (cellule comprise, en approvisionnement), était peu profond (réf. N562, Figure 1E). Le nid illustré à la Figure 1F (réf. N563) était en cours de creusement. Sa galerie principale, d'abord presque verticale, part soudain en oblique, probablement parce que la fondatrice a rencontré un caillou dans sa progression souterraine. Le dernier nid trouvé (réf. N564, Figure 1G) est un court forage de $8 \mathrm{~mm}$, en butée sur un caillou et qui servait momentanément d'abri à la femelle que j’y ai trouvée. Nul doute que cette petite cavité aurait été abandonnée pour un nouveau forage.

Dans le même secteur, j'avais localisé d'autres nids et placé des repères au sol pour les retrouver le lendemain. De retour sur les lieux, certaines ouvertures n'étaient plus visibles : j'ai ainsi pu constater que les nids étaient obturés par un cloisonnement de terre prise sur place et 

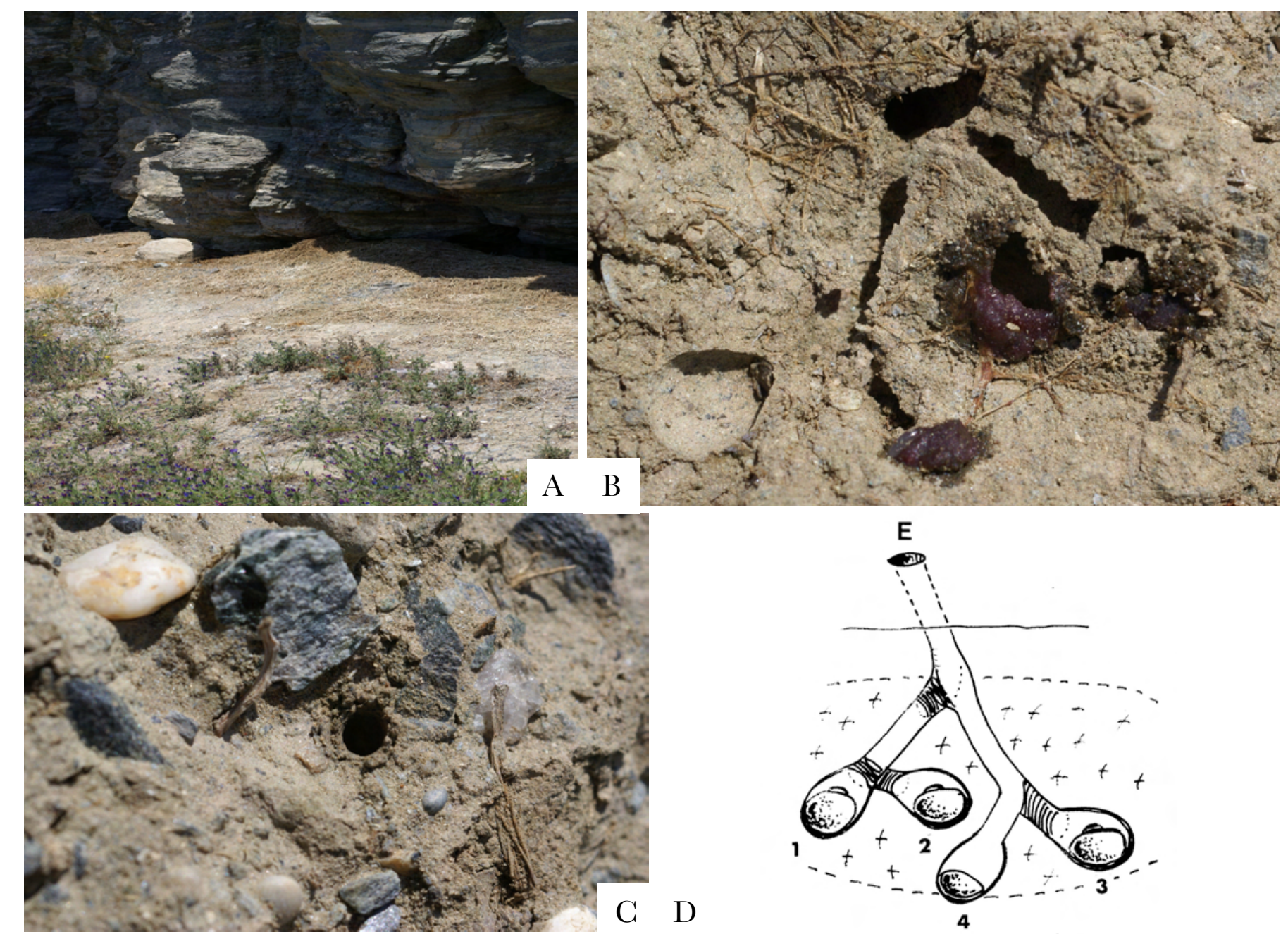

E $\quad \mathrm{F}$

G
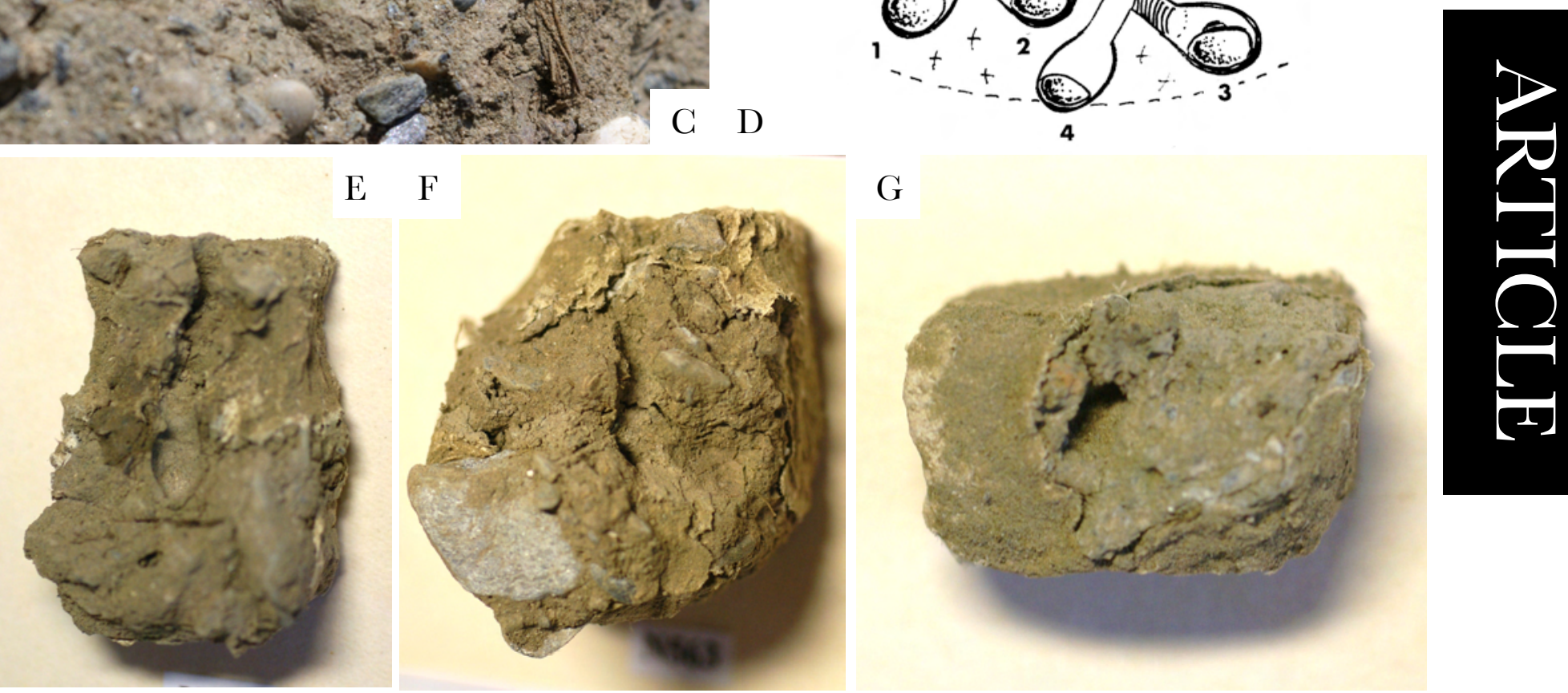

Figure 1. Détail des nids souterrains d'Hoplitis (Annosmia) annulata subsp. annulata LATREILLE (Photos et illustration G Le Goff).

qui, une fois sec et plus ou moins couvert de poussière, ne se distinguait plus du substrat environnant (sur mes schémas, les nids n'étant pas achevés, je n'ai pas figuré ce bouchon final).

A noter que l'entrée du nid, avant son achèvement, est consolidée et de diamètre légèrement rétréci par rapport à celui de la galerie même (Figure 1C), laissant dès lors juste le passage de la fondatrice et offrant peutêtre une meilleure défense contre les intrus parasites.

\section{H. (A.) annulata ssp. corsaria WARNCKE}

Cette sous-espèce a une distribution limitée à la Corse. Le matériel décrit ci-dessous a été récolté à Porticcio (Corse, France) et est conservé dans ma collection personnelle (Nid N476 - fondatrice $N^{\circ} 10430$ - collecte du 16.vi.03). Les visites florales ont été étudiées localement sur Echium plantagineum L. (Boraginaceae) (Figure 2). 

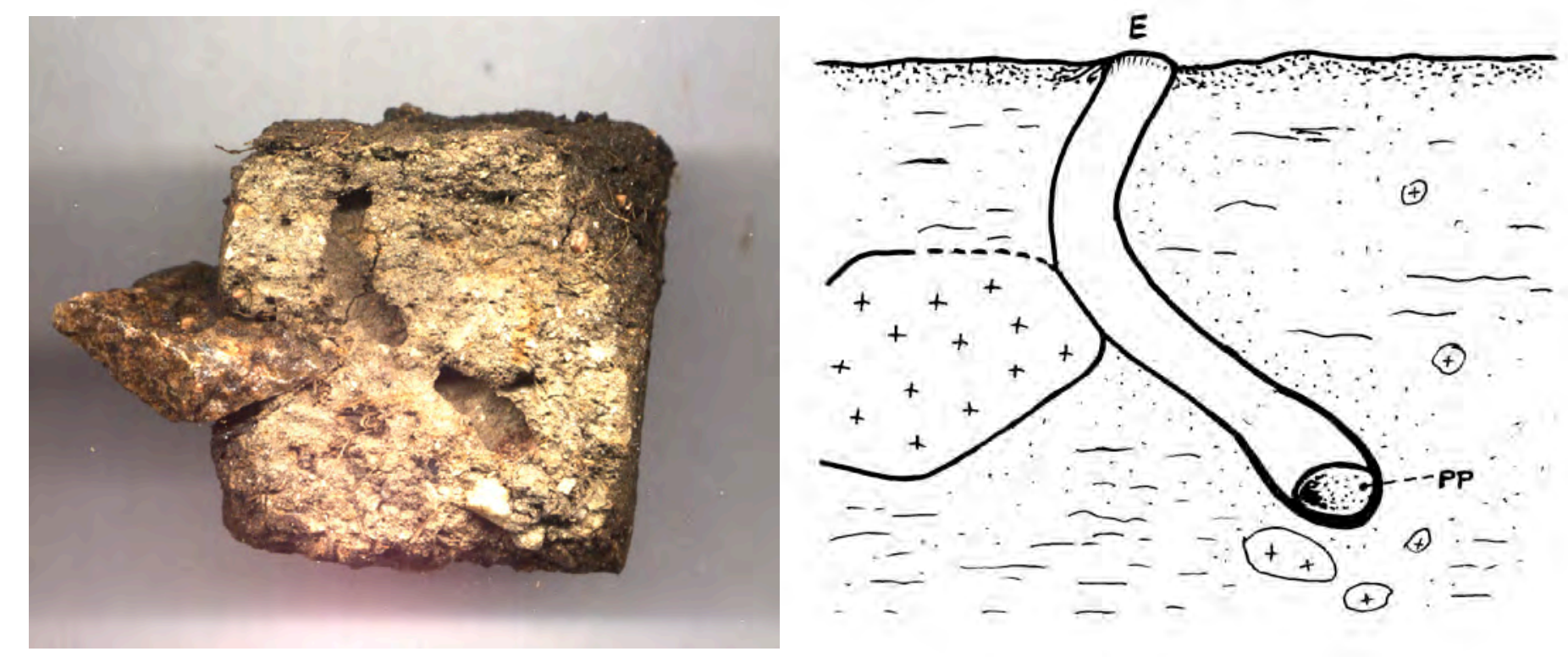

Figure 2. Détail des nids souterrains d'Hoplitis (Annosmia) annulata subsp. corsaria WARNCKE (Photo et illustration G Le Goff).

J'ai collecté plusieurs mâles dans l'environnement immédiat du nid. Ce sont eux qui m’ont permis d'identifier la sous-espèce de Warncke (1991) : leur principale caractéristique est la ponctuation éparse et fine sur une grande partie des joues (« comme des trous d'épingle » -cf. Warncke (1991)), alors qu'elle est serrée et profonde chez la ssp. précédente.

La femelle d'H. (A.) annulata corsaria WARNCKE ressemble beaucoup à l'espèce nominative. La ponctuation des tergites est cependant plus fine et moins profonde. La zone brillante clypéale forme une petite tache triangulaire ; celle de l'espace inter-antennaire est presqu'ovale. Le haut de cet espace est légèrement bosselé avant le front avec un point particulièrement brillant. Suit, une courte ligne brillante qui s'étend sur $1 / 4$ de la ligne médiane, entre l'espace inter-antennaire et l'ocelle antérieur. Chez annulata annulata, le clypéus est en grande partie brillant (large triangle suivi d'une ligne médiane atteignant l'apex). La brillance est triangulaire entre les antennes et se prolonge en longue pointe devenant linéaire sur le front, occupant $3 / 4$ de la distance vers l'ocelle unique et ceci dans un plan quasi horizontal. Chez annulata crenulata, la brillance frontale est limitée à une tache suivant l'espace inter-antennaire.
Les franges de poils apicales des tergites sont plus étroites chez annulata et corsaria que chez crenulata.

J'ai collecté l'unique nid (réf. N476) (Figure 2) sur les hauteurs de Porticcio, dans un terrain herbeux sec où poussent quelques pieds ras d'Echium plantagineum. Comme pour la ssp. précédente, la galerie commence verticalement puis part en oblique quand la fondatrice a rencontré une pierre dans son forage. La galerie longue de $30 \mathrm{~mm}$ mène à une cellule unique en approvisionnement. Comme toujours c'est un Echium qui fournit le pollen nourricier.

\section{H. (A.) annulata ssp. crenulata MORAWITZ}

Gette sous-espèce a une distribution méditerranéenne moyenne et orientale. Le matériel décrit ci-dessous a été récolté en Crète (Grèce) et est conservé dans ma collection personnelle (Nid N565 fondatrice $\mathrm{N}^{\circ} 13271$ - collecte du 17/06/07 - Adele (N. Reth.) - Crète - butinage d'Echium angustifolium - Figure 3 ; Nid réf. N520 - fondatrice $\mathrm{N}^{\circ} 12303$ - collecte du 16.vi.05 - Gra Ligia (N. Lassith.) - Crète - butinage d'E. angustifolium - Figure 4).
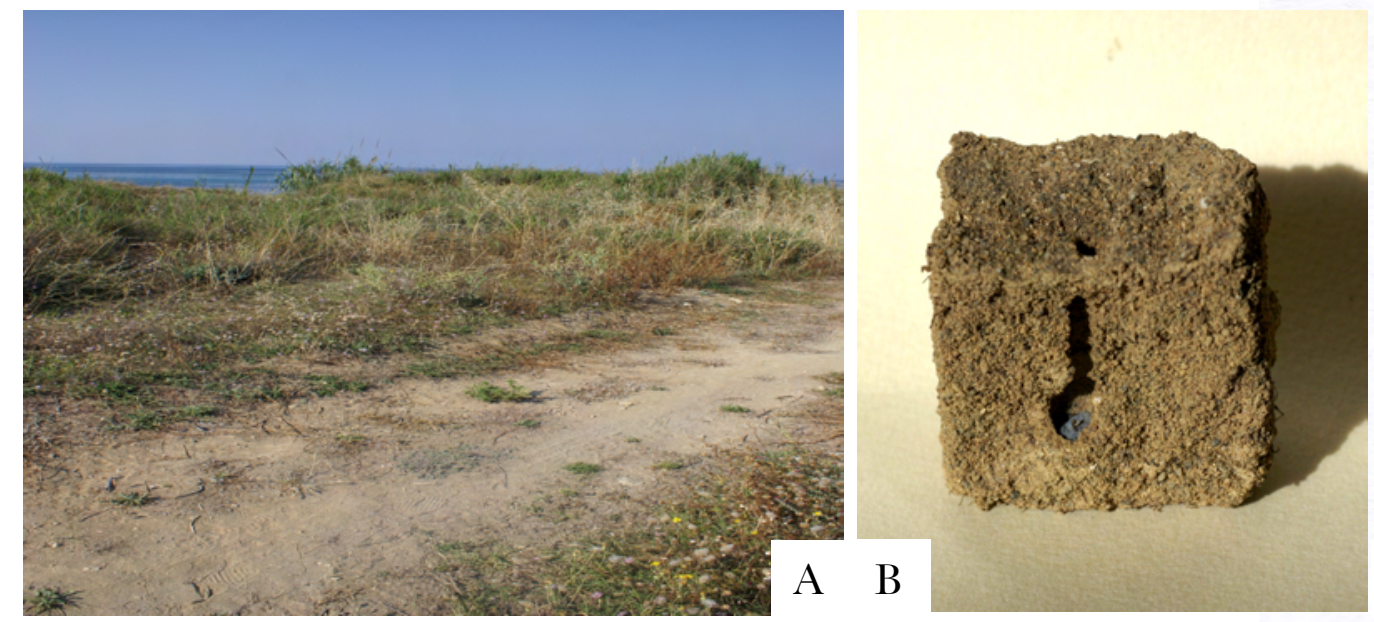

$E$

Figure 3. Détail des nids souterrains d'Hoplitis (Annosmia) annulata subsp. crenulata MoRAWITz (Photos et illustration G Le Goff). 

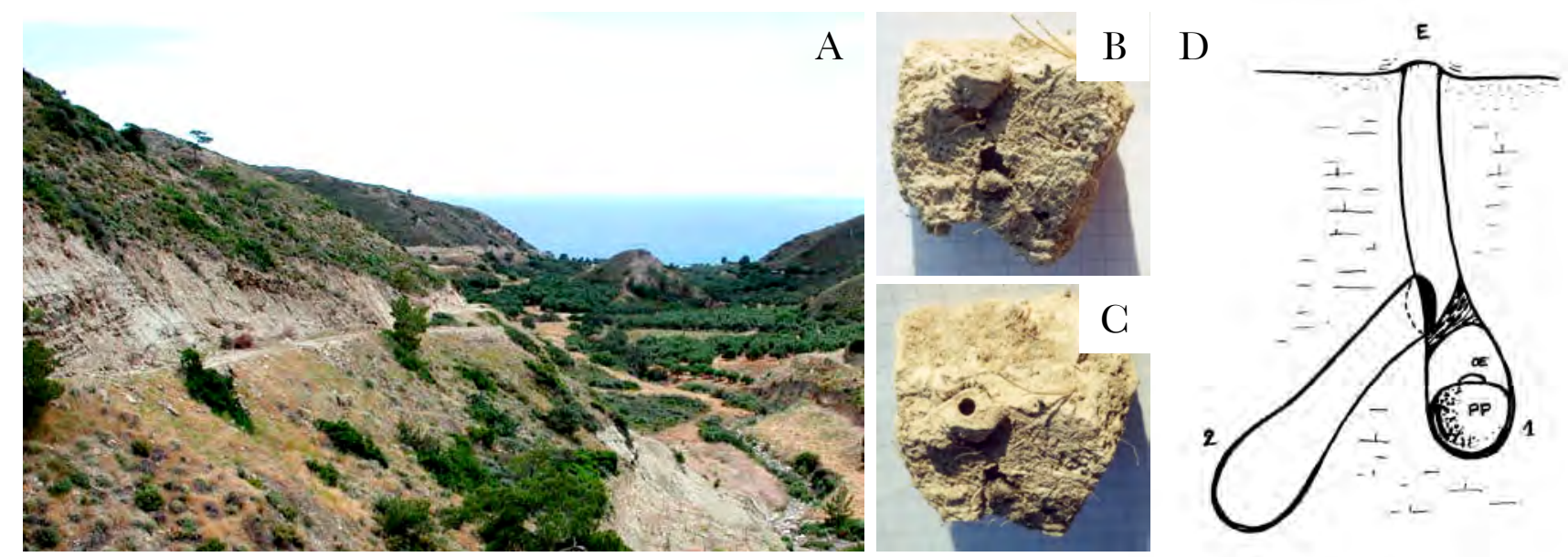

Figure 4. Détail des nids souterrains d'Hoplitis (Annosmia) annulata subsp. crenulata MORAWITz (Photos et illustration G Le Goff).

J'ai collecté le nid réf. N565 au bord d'un chemin près de la mer, entre un champ de luzerne et la zone dunaire donnant sur la plage (Figure 3A). Des touffes d'E. angustifolium poussent çà et là dans cette zone des Kampos. Les $H$. annulata crenulata sont en nombre à butiner ces zones où fleurissent les Echium. Le nid était creusé verticalement dans le sol de terre sableuse. L'ouverture était sous le couvert d'une touffe d'herbe. Le forage est très court : $22 \mathrm{~mm}$ (cellule comprise). La loge était approvisionnée, avec l'oeuf sur la pâtée violette (Figure 3B-G). Les cloisonnements restaient à faire au moment de la collecte.

J'ai trouvé l'autre nid (réf. N520, Figure 4) au sud-est de la Crète sur le bord d'une piste étroite taillée au flanc d'un versant, au-dessus d'un ruisseau (Figure 4A). Le sol est argilo-calcaire. Le nid était à découvert, entre deux touffes courtes d'E. angustifolium. Il était composé d'une première galerie avec une cellule complète. A partir du cloisonnement biaisé de cette cellule, une $2^{\text {ème }}$ galerie, oblique, un peu plus courte que la première, mène à une loge en construction (Figure 4D). Sur ce nid, on voit bien le léger rétrécissement consolidé de l'ouverture (Figure 4C-D-.

\section{H. (A.) tkalcuella LE GoFF}

La distribution de cette espèce est limitée à la Péninsule Ibérique. Le matériel décrit ci-dessous a été récolté à Guardamar del Segura (Alicante, Espagne) et est conservé dans ma collection personnelle (Nid réf. N415 - fondatrice No8796 (Allotype) - collecte du 18.v. 01 - butinage d'E. plantagineum - Figure 5)

J'ai collecté le bloc de terre contenant le nid après la capture de la femelle fondatrice et des parasites qui s'introduisaient dans le trou d'accès (Mutilidae : Smicromyrme partita (KLUG) et Chrysididae : Chrysura hybrida LEPELETIER). Je pensais simplement avoir affaire à $H$. annulata annulata. Ce n'est qu'au retour de mon séjour que j'ai dégagé le nid en question. En examinant un imago mâle, j'ai constaté être en présence d'une espèce nouvelle. Pour confirmation, j’ai contacté B. Tkalcu à qui je souhaitais dédier cette Annosmia. Cette nouvelle Hoplitis a fait l'objet d'un article en 2003 (voir Le Goff 2003). Les types mâle et femelle, ont été offerts à la Dra Conception Ornosa Gallego et sont déposés à l'Université Complutense de Madrid. Un paratype mâle a été déposé au MNHN (Paris). L'espèce est désormais classée dans le groupe d'H. zonalis (Müller, 2010).

Le nid, comme chez les précédentes Annosmia, était dans le sol. Le forage initial forme une galerie d'accès très courte qui se ramifie ensuite en quatre branches; le nid comprend 9 cellules (Figures 5C-F). Chaque loge est une coque ovale de terre malaxée durcie, parfaitement lissée intérieurement ; un bouchon fait de couches successives sépare les cellules entre elles; le même procédé est utilisé pour clore et obturer l'ouverture du nid. La provision est là aussi semi-liquide et farinée du pollen d'E. plantagineum qui poussait au pied du talus. Dans le nid se trouvaient simultanément une cellule en cours d'approvisionnement, 2 imagos et 6 larves matures. Pour l'anecdote, cette zone de découverte a complètement été rasée : la Huerta, avec ses plantations et ses jardins ouverts, a laissé place à des immeubles et des tours (voir Figure 5A). Heureusement j’ai retrouvé l'année suivante la petite Hoplitis dans un secteur archéologique protégé dans la pinède de Guardamar del Segura.

\section{Conclusion sur la nidification de ce groupe d'Osmiini}

Ces quelques exemples montrent que les Hoplitis du sbg. Annosmia nidifient dans le sol en creusant ellesmêmes leur nid. Chaque cellule est de forme ovale ; la paroi de la loge est durcie par du mucus et parfaitement lissée. La provision semi-liquide est toujours élaborée à partir du pollen de différentes espèces d'Echium. Chaque cellule est cloisonnée. Si les nids unicellulaires ont été les premiers décrits et apparaissent les plus nombreux, plusieurs des nids décrits plus haut montrent l'existence de nids pluricellulaires, allant jusqu'à 9 cellules chez $H$. tkalcuella. Le forage en sol favorable est bien vertical, plus ou moins profond, mais il se trouve dévié obliquement à la rencontre d'obstacles naturels, comme les cailloux par exemple, ou des cloisonnements déjà établis (forage d'une autre galerie dans les nids pluricellulaires). Le nid est finalement obturé, sans aucune trace visible à la surface du sol. 


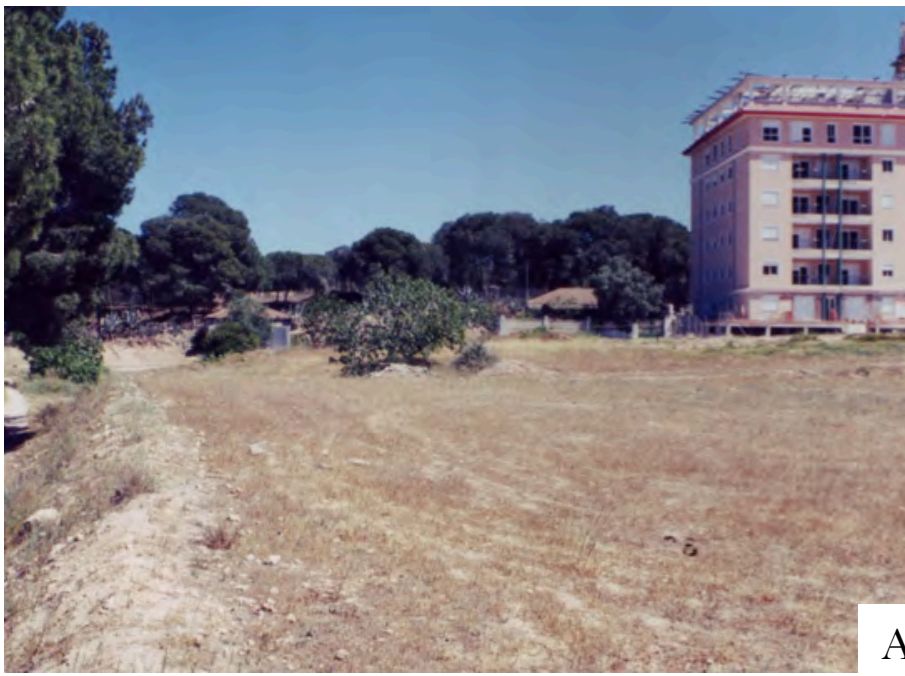

G

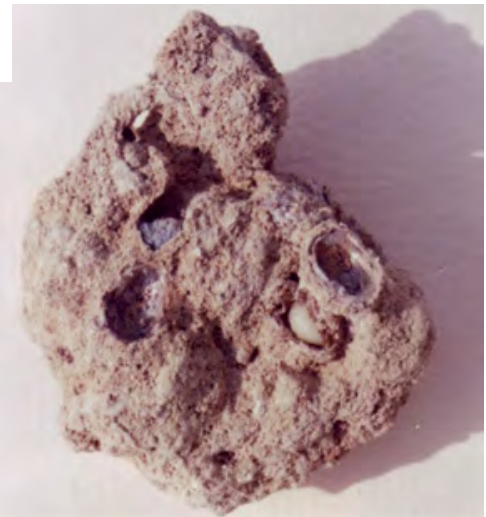

$\mathrm{D}$

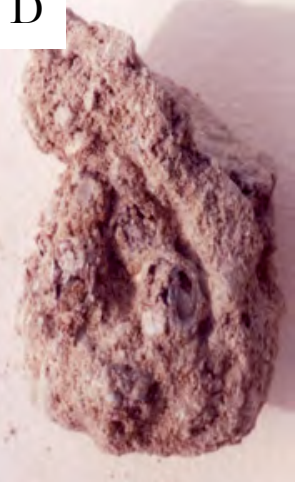

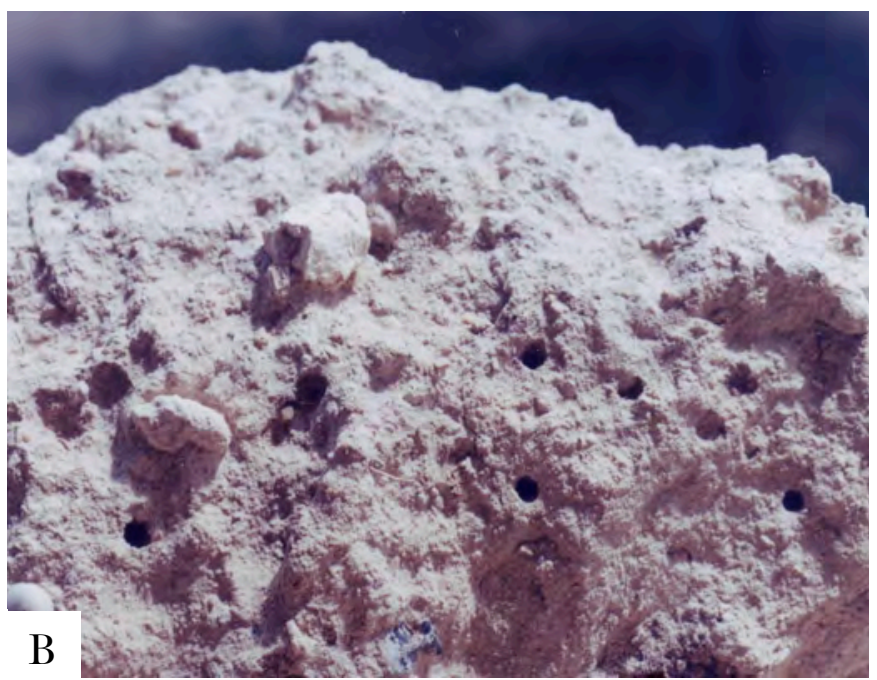

$\mathrm{E}$

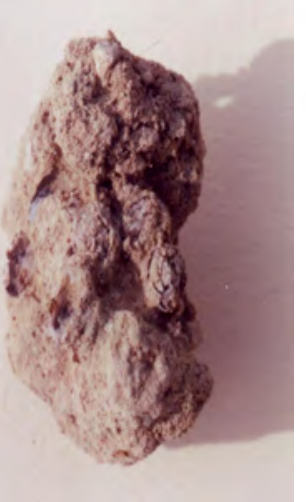

$\mathrm{F}$

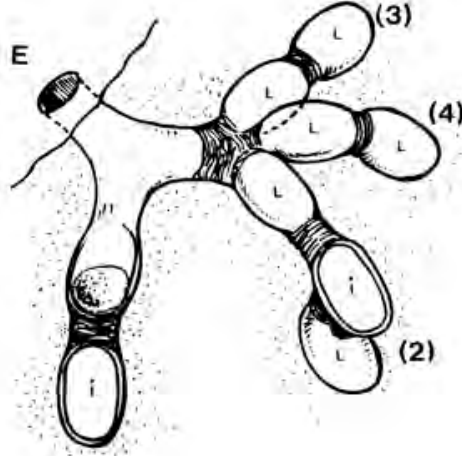

(1)

Figure 5. Détail des nids souterrains d'Hoplitis (Annosmia) tkalcuella LE GoFF (Photos et illustration G Le Goff).

\section{Références bibliographiques}

Benoist R, 1929. Bulletin de la Société Entomologique de France 34 : 99.

Benoist R, 1934. Bulletin de la Société Entomologique de France 39 : 106

Griswold TL \& Michener CD, 1998. The classification of the

Osmiini of the eastern hemisphere. Fournal of the Kansas

Entomological Society $70: 207-253$.

Ferton G, 1893. Sur les moeurs de quelques hyménoptères de la Provence. Actes de la Société Linnéenne de Bordeaux: Tome XLV : 11.

Latreille P-A, 1811. Encyclopédie Méthodique des Insectes $8: 587$

Le Goff G, 2003. Une nouvelle espèce ibérique du genre Hoplitis

KLUG (Hymenoptera, Apoidea, Megachilidae). Bulletin de la Société Entomologique de France 108(1) : 67-70.

Mavromoustakis GA, 1949. Eos 25 : 289-290, 292-293

Mavromoustakis GA, 1954. New and interesting bees from Israel. Bulletin of the Research Concil of Israel 4 : 263-266.

Michener CD, 2007. The Bees of the World, second edition. The Johns Hopkins University Press, Baltimore.

Morawitz F, 1871. Horae Societatis Entomologicae Rossicae 8 : 208-209.

Morawitz F, 1877. Horae Societatis Entomologicae Rossicae 14 : 40-42.

Müller A, 2010. Palaearctic Osmiïne Bees. ETH Zürich, blog consulté le 10.x.2010 à l'adresse http://blogs.ethz.ch/osmiini
Pérez J, 1895. Nouvelles Espèces de Mellifères de Barbarie : p.14

Stanek E, 1969. Nachr. Naturwiss. Mus. Aschaffenburg 78 : 11-12.

Ungricht S, Müller A \& Dorn S, 2008. A taxonomic catalogue of the Plaearctic bees of the tribe Osmiini (Hymenoptera : Apoidea : Megachilidae). Zootaxa 1865 (sept. 2008).

Warncke K, 1977. Ideen zum natürlichen System der Bienen (Hymenoptera, Apoidea). Mitt. Münch. Ent. Ges. 67 : 39-63

Warncke K, 1991. Die Bienengattung Osmia PANZER, 1806, irhe Systematik in der West-paläarktis und ihre Verbreitung in der Türkei. 9. Die Untergattung Annosmia subg. n. Linzer Biologische Beiträge 23(1) : 307-336.

Warncke K, 1991. Die Bienengattung Bytinskia Mavromoustakis (Hymenoptera, Apidae, Megachilidae). Linzer Biologische Beiträge 23(2): 483-489.

Zanden G van der, 1991b. Neue Arten der Palärktischen Osmiini und einige neue Fälle von Synonymie (Hymenoptera aculeata, Apoidea, Megachilidae). Linzer Biologische Beiträge 23(1) : 345-363.

Zanden G van der, 1991c. Neue oder wenig bekannte Arten der Osmiini aus dem paläarktischen Gebiet (Insecta, Hymenoptera, Apoidea : Megachilidae). Reichenbachia 28(31): 163-171.

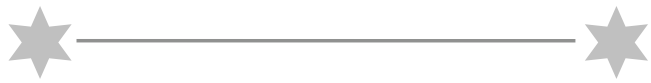

